\title{
Long COVID - the physical and mental health of children and non-hospitalised young people 3 months after SARS-CoV-2 infection; a national matched cohort study (The CLoCk) Study.
}

Terence Stephenson ( $\nabla$ t.stephenson@ucl.ac.uk) UCL Great Ormond Street Institute of Child Health

\section{Terence Stephenson}

UCL Great Ormond Street Institute of Child Health

\section{Snehal Pinto Pereira}

Division of Surgery \& Interventional Science, University College London

\section{Roz Shafran}

UCL Great Ormond Street Institute of Child Health

\section{Bianca De Stavola}

UCL Great Ormond Street Institute of Child Health

\section{Natalia Rojas}

UCL Great Ormond Street Institute of Child Health

\section{Kelsey McOwat}

Immunisation Department, Public Health England, 61 Colindale Avenue, London

\section{Ruth Simmons}

Immunisation Department, Public Health England, 61 Colindale Avenue, London

\section{Maria Zavala}

Immunisation Department, Public Health England, 61 Colindale Avenue, London

\section{Lauren O'Mahoney}

Diabetes Research Centre, University of Leicester, Leicester General Hospital

\section{Trudie Chalder}

Kings College London

\section{Esther Crawley}

Centre for Academic Child Health, Bristol Medical School, University of Bristol

\section{Tamsin Ford}

Department of Psychiatry, University of Cambridge

\section{Anthony Harnden}

Nuffield Department of Primary Care Health Sciences, University of Oxford Isobel Heyman

UCL Great Ormond Street Institute of Child Health 


\section{Olivia Swann}

Department of Child Life and Health, University of Edinburgh, UK

\section{Liz Whittaker}

Centre for Paediatrics and Child Health, Imperial College London

\section{CLoCk Consortium CLoCk Consortium}

Various - listed in manuscript

Shamez Ladhani

Public Health England

\section{Article}

Keywords: Long COVID, children and young people, matched cohort study, physical health, mental health

Posted Date: August 10th, 2021

DOl: https://doi.org/10.21203/rs.3.rs-798316/v1

License: (c) (i) This work is licensed under a Creative Commons Attribution 4.0 International License.

Read Full License 


\section{Abstract}

Introduction: We describe post-COVID symptomatology in a national sample of 11-17-year-old children and young people (CYP) with PCR-confirmed SARS-CoV-2 infection compared to test-negative controls.

Methods and analysis: A cohort study of test-positive $(n=3,065)$ and age-, sex- and geographicallymatched test-negative CYP $(n=3,739)$ completed detailed questionnaires 3 months post-test.

Results: At PCR-testing, 35.4\% of test-positives and $8.3 \%$ of test-negatives had any symptoms whilst $30.6 \%$ and $6.2 \%$, respectively, had $3+$ symptoms. At 3 months post-testing, $66.5 \%$ of test-positives and $53.3 \%$ of test-negatives had any symptoms, whilst $30.3 \%$ and $16.2 \%$, respectively, had $3+$ symptoms. Latent class analysis identified two classes, characterised by "few" or "multiple" symptoms. This latter class was more frequent among test-positives, females, older CYP and those with worse pre-test physical and mental health.

Discussion: Test-positive CYP had a similar symptom profile to test-negative CYP but with higher prevalence of single and, particularly, multiple symptoms at PCR-testing and 3 months later.

\section{Introduction}

Children and young people (CYP) are more likely to have a mild illness and less likely to be hospitalised following SARS-CoV-2 exposure compared to adults. More children ${ }^{1}$ recover without sequelae compared to adults ${ }^{2}$. However, we know little about the diagnosis, prevalence, phenotype or duration of Long COVID (also called long haulers and post-acute COVID syndrome) in CYP. More than 200 different symptoms have been associated with Long $\operatorname{COVID}^{3,4}$, with an adult prevalence up to $80 \%^{5}$. Long COVID has been reported in those symptomatic or asymptomatic at time of SARS-CoV-2 testing, and in laboratoryconfirmed or unconfirmed cases, with symptoms beginning at or after acute infection, which may be persistent, intermittent or relapsing in nature ${ }^{3,5}$. Adolescents may have a higher risk than younger children ${ }^{1,6}$ but it is unclear whether the features associated with Long COVID are related to the viral infection or the effects of the pandemic, lockdown and school closures with consequent social isolation.

A literature review of Long Covid in CYP identified 17 relevant publications with sample size $\geq 10$ (Supplementary Table 1). Existing studies were all observational, including cohort $(n=9)$, matched cohort $(n=2)$, and cross-sectional $(n=6)$ designs. Clinical diagnosis of COVID-19 infection was confirmed by either a positive reverse transcription polymerase chain reaction (PCR) result or serological testing in 13 studies; the remaining studies included COVID-19 cases that were diagnosed clinically, were self-reported COVID-19, using rapid antigen tests or through an unspecified method of confirmation. These studies included 15,250 CYP up to 20 years with follow-up of 28 to 324 days. The most common persistent symptoms across studies were fatigue, insomnia, anosmia and headaches. The prevalence of Long 
COVID in CYP with laboratory-confirmed or suspected COVID-19 ranged from $1 \%-51 \%$, with smaller studies reporting higher prevalence rates.

An online, nationally-representative survey of 313,216 people asking about self-reported Long COVID (unexplained symptoms persisting for more than four weeks after suspected COVID-19) estimated the UK population Long COVID prevalence to be $0.14 \%$ for $2-11$ years, $0.50 \%$ for $12-16$ years, and $1.51 \%$ for $17-24$ years ${ }^{6}$.

The mental health of CYP have received less attention than the physical symptoms of COVID-19, despite the significant negative impact of the pandemic on their wellbeing ${ }^{7}$. Miller, et al. ${ }^{8}$ found $10 \%$ of children with Long COVID reported unspecified persistent psychological/psychiatric symptoms. However, no significant differences between depression, anxiety, and perceived stress were found comparing CYP with Long COVID to COVID negative controls ${ }^{9}$. Similarly, a study of 1,560 CYP (median age 15 years) found a high rate of neurocognitive, pain and mood symptoms but no difference between seronegative and seropositive participants, highlighting the importance of including a negative control group in longitudinal studies of Long COVID ${ }^{10}$.

The CLoCk study is a national, longitudinal cohort study of CYP in England ${ }^{11}$ with the primary aim of describing the clinical phenotype and prevalence of post-COVID physical symptoms and mental health problems among CYP with laboratory-confirmed SARS-CoV-2 infection compared to test-negative controls, to identify those most at risk and their illness trajectory. This paper presents the results of the study 3 months after PCR-testing in CYP across England.

\section{Methods}

\section{Design}

A cohort study of SARS-CoV-2 PCR-positive CYP aged 11-17 years matched on month of test, age, sex and geographical area to SARS-CoV-2 test-negative controls selected from the database of test results held by Public Health England (PHE). Since the start of the pandemic in England, PHE receives daily electronic notifications of all SARS-CoV-2 PCR tests performed in healthcare settings (Pillar 1 tests) and in the community (Pillar 2 tests) reported through the Second Generation Surveillance System (SGSS). Information within the SGSS includes National Health Service (NHS) number, name, age, sex, postcode, date of sample, reporting laboratory and test result. PHE also has access to the electronic Patient Demographic Service (PDS), which contains the names, postal addresses and vital status (alive/dead) of all patients registered with the NHS. 


\section{Participants}

Between September 2020 and March 2021, 234,803 young people aged between 11 and 17 years tested positive for SARS-CoV-2 in England. During the same period, there were 1,481,154 negatives tests among this age-group from 1,203,996 CYP (some CYP had more than one negative test). Among those who tested negative, 76,689 individuals $(100,154$ tests) were excluded as they had a positive result before or (up to 31 March 2021) after their negative test. The 234,803 test-positive CYP were matched with some oversampling to negative CYP according to their age at test, gender, month of test, and lower super output area (geographical area of $~ 1500$ people), resulting in 102,402 test-positive individuals and 147,561 matched, negative individuals. Using secure procedures, matched individuals were checked against the NHS PDS to exclude individuals who had died and to extract participants' postal addresses. 37 individuals were excluded because they had died since their COVID-19 test (6 test-positives, 31 testnegatives), while 11,193 test-positive individuals and 19,251 test-negative individuals were excluded because a residential address was not available. Finally, 246 young people were excluded because they were included in a previous pilot study. Following these exclusions, 91,016 test-positive CYP and 128,220 negative CYP were contacted. A letter was posted to all those selected, inviting them to take part in this study using an online link which provided them with details of the study, an option to consent online and complete a short recruitment questionnaire.

We began contacting individuals from April 2021 onwards. In this paper, we focus on those who were tested in January-March 2021 because only they could report symptoms 3 months post-test with minimal recall bias of symptoms at time of testing. For this group, a total of 50,846 individuals (23,048 testpositives, 27,798 test-negatives) were invited to participate (Fig. 1).

\section{Sample size calculations}

The original study design was based on the calculation that 5,000 participants (2,500 test-positives, 2,500 test-negatives) would have $80 \%$ power to detect at least a $4 \%$ difference in symptom frequency at $5 \%$ significance, if test-negative participants had a $34 \%$ prevalence (based on available data at the time from the sKIDs study ${ }^{12}$ ), accounting for attrition and possible lower baseline symptom prevalence. However, studying multiple symptoms and identifying risk factors for Long COVID requires a larger sample size. For this reason, we amended our calculations to invite all available participants in England (except those tested in December 2020 due to funding constraints at present) ${ }^{11}$.

\section{Data collection}

Participants who were tested between January-March 2021 were contacted 3 months after testing. Following online informed consent, the CYP self-completed an online (or paper) questionnaire about their physical and mental health at the time of the original test ("baseline") and at the time of completing the questionnaire; younger CYP and CYP with special educational needs or disability could request the help of their carer. The completed questionnaires were returned at a median time of 14.9 weeks after testing 
[25th ,75th centiles: 13.1, 18.9]. A total of 63 test-negative CYP reported having had a previous positive SARS-CoV-2 test and were excluded from analysis.

\section{Measures}

The first questionnaire sent to CYP included demographic characteristics, elements of the International Severe Acute Respiratory and emerging Infection Consortium (ISARIC) Paediatric COVID-19 follow-up questionnaire ${ }^{13}$ and the recent Mental Health of Children and Young people in England surveys (https://tinyurl.com/NHSWave1FU). The follow-up questionnaires were identical but did not include questions on demographic characteristics. They were designed together with ISARIC Paediatric Working Group to produce a harmonised data collection tool, to facilitate international comparisons regarding the risk factors and profile of Long COVID in CYP.

The elements taken from the ISARIC Paediatric COVID-19 follow-up questionnaire ${ }^{13}$ included questions about physical symptoms, particularly cough and fever (the main acute symptoms in non-hospitalised CYP $)^{14}$ and gastrointestinal symptoms which were commonly reported in seropositive CYP ${ }^{15}$. Other symptoms which might manifest later in Long COVID (e.g., tiredness, headaches, myalgia etc.) were also included.

We asked CYP to rate their general physical and mental health before their SARS-CoV-2 test, in two separate questions using a 5 category Likert scale; in analyses we recoded these variables into two categories (very poor/poor/ok versus good/very good). To measure mental health and wellbeing, the Strengths and Difficulties Questionnaire (SDQ $)^{16}$ was summarised into the total difficulties score that excluded the prosocial dimension, along with the short 7-item version of the Warwick Edinburgh Mental Wellbeing Scale (SWEMWBS) ${ }^{17}$. A higher SDQ total difficulties score is indicative of more problems, whereas a higher SWEMWBS score indicates a higher level of mental well-being. Quality of life/functioning was measured via the EQ-5D-Y ${ }^{18}$ and fatigue was measured by the 11 -item Chalder Fatigue Questionnaire (CFQ) ${ }^{19}$.

\section{Statistical methods}

To assess the representativeness of our study participants we compared their demographic characteristics (sex, age, region of residence) to those of the target population. The participants' demographic characteristics, physical symptoms at "baseline", and physical symptoms, mental health status, well-being, quality of life/functioning, and fatigue 3-months post-test were compared by SARSCoV-2 test status. We carried out comparisons separately by age-groups (11-15y vs. 16-17y) as the prevalence of Long COVID may vary by age (https://tinyurl.com/ONSPrevalence0721).

We used latent class analysis ${ }^{20}$ to assess whether and how baseline and 3-month physical symptoms clustered among CYP, allowing for differential model parametrization by SARS-CoV-2 test status (while analysing the data jointly by test status but separately by time). The number of classes was selected by comparing the Bayesian Information Criteria. Predicted class membership was estimated and used to 
assign CYP to their most likely class; this classification was then used to describe the characteristics of the latent classes.

As this is mainly a descriptive study, we do not report p-values for comparisons by SARS-CoV-2 test status. We do report estimates of latent class prevalence by SARS-CoV-2 test status, as well as their ratio, with confidence intervals computed using the delta method ${ }^{21}$. To assess the impact of potential response bias, we reweighted all symptom frequencies according to the age, sex, region and SARS-CoV-2 test status of the responders.

\section{Results}

Study representativeness

A total of 6,804 CYP who had been tested between January and March 2021 participated in the study by completing the 3-month questionnaire. The overall response rate was $13.4 \%$, with a similar proportion of test-positives (13.3\%) and test-negatives (13.5\%) contributing (Table 1). More females and older CYP (1617-year-olds) responded. Response rates also varied by region of England. Overall, there was little difference in demographic characteristics between test-positive and test-negative participants, reflecting the matched study design (Table 2).

Physical symptoms and profile: baseline and 3-month post-test

At the time of testing, test-positive CYP had higher percentages of physical symptoms compared to testnegative CYP (Table 3 ); $35.4 \%$ of test-positives and $8.3 \%$ of test-negatives had any symptoms whilst $30.6 \%$ of test-positives and $6.2 \%$ of test-negatives had $3+$ symptoms. The types of symptoms reported by test-positives and negatives were the same in the two age-groups: the most common symptoms among test-positives were sore throat, headache, tiredness and loss of smell while test-negatives had sore throat, headache, fever and persistent cough. The prevalence of these symptoms, however, varied by SARS-CoV2 test result (e.g. $26.3 \%$ of positives compared to $4.8 \%$ of negatives reported headaches).

Three months after the SARS-CoV-2 test, the presence of physical symptoms was higher than at baseline in both groups; $66.5 \%$ of test-positives and $53.4 \%$ of test-negatives had any symptoms whilst $30.3 \%$ of test-positives and $16.2 \%$ of test-negatives had $3+$ symptoms. The symptom profile did not vary by age: for both 11-15y and 16-17y the most common symptoms among test-positives were tiredness, headache and shortness of breath and, among test-negatives, tiredness, headache and the unspecified category of "other". Again, the prevalence of tiredness and headache was consistently higher in the test positives, $39.0 \%$ and $23.2 \%$ versus $24.4 \%$ and $14.2 \%$ in negatives, respectively. Prevalence was higher for $16-17$ - 
year-olds; for example, $46.4 \%$ of test-positives reported being tired compared to $29.6 \%$ of test-negatives. When we reweighted the percentage of reported symptoms at baseline and at 3 months post-test, broadly similar patterns were observed to those reported above (Supplementary Table 2).

Mental health, well-being, quality of life/functioning and fatigue 3-month post-test

There was no difference in the distribution of mental health scores (assessed by the SDQ total difficulties scores) and well-being (assessed by SWEMBS) between test positives and negatives, overall or in either age-group. The SDQ median $\left(25^{\text {th }}, 75^{\text {th }}\right.$ centile) was $10(6,15)$ for both test-positive and test-negative CYP aged 11-15y. For CYP aged 16-17y, the corresponding values were $11(7,16)$ for test-positives and 12 $(8,16)$ for test-negatives. Likewise, SWEMBS scores were similar among test-positives (Mean=21.5, $S D=4.3$ ) and test-negatives (Mean=21.4, SD=4.3). Similarly, fatigue (assessed by CFQ) showed no substantial differences between positives (Mean=13.3, $S D=5.2$ ) and negatives (Mean=12.5, SD=5.1). However, older CYP (16-17y) did report slightly higher values: test-positives (Mean=14.0, SD=5.5) and test-negatives (Mean=13.4, SD=5.2). In terms of Health-Related Quality of Life (EQ-5D-Y) test positives in both age groups were more likely to report problems with mobility, doing usual activities, and pain/discomfort (Supplementary Figure 1). Strikingly, while $40.8 \%$ of positives felt worried, sad or unhappy on the single item of the EQ-5D-Y, 39.2\% of the negatives also reported feeling this way (Supplementary Figure 1).

Physical symptom clustering at baseline and 3-months post-test

No evidence of clustering of baseline symptoms was found for either test-positive or test-negative participants. There was, however, evidence of clustering in symptoms reported at 3 months, with two subgroups emerging for both test-positive and test-negative CYP (Figure 2). In each, the largest subgroup (class 1 ) had very low prevalence of most symptoms, while the second subgroup (class 2 ) was characterised in both positives and negatives by multiple symptoms dominated by tiredness, headache, shortness of breath and dizziness. We refer to these classes as "few" and "multiple" symptoms classes. The estimated probability (risk) of being in the multiple symptom class (class 2 ) was $29.6 \%(95 \%$ confidence interval, $27.4 \%, 31.7 \%)$ for test-positives and $19.3 \%(17.7 \%, 21.0 \%)$ for test-negatives and the risk ratio of being in class 2 versus class 1 comparing test-positives to test-negatives was $1.53(1.35$, 1.70). 
For both test-positive and test-negative CYP, those assigned to class 2 were more likely to be female, older, to have poorer baseline physical and mental health (relative to the overall percentages of $19 \%$ and $30 \%$ ) and, at 3-months, to be more likely to have problems with mobility, self-care, usual activities and pain/discomfort. They also have higher SDQ total difficulties and CFS scores, and lower SWEMBS scores (Table 4).

\section{Discussion}

Given that there is no universally agreed definition of Long COVID, we elected not to start with an arbitrary definition of a new condition but to seek the descriptions from almost 7,000 CYP of their physical and mental experiences in the months following a SARS-CoV-2 test. This is the largest study on symptoms post-COVID in children to date and, uniquely, uses child-reported symptoms, is confined to PCR-proven SARS-CoV-2 status, has a PCR-negative control group, and uses standardised measures to assess mental health, well-being, and fatigue.

Several important findings contribute to the current knowledge of Long COVID in CYP. First, three months after the SARS-CoV-2 test, the presence of physical symptoms was higher than at the time of testing. This finding emphasises the importance of having a comparison group to objectively interpret the findings and derive prevalence estimates. Although $64.6 \%$ of test-positives reported no symptoms at time of testing (compared to $91.7 \%$ of test-negatives), they did not continue to remain asymptomatic, with only $33.5 \%$ of test-positives (and $46.7 \%$ of test-negatives) reporting no symptoms at 3 months. This finding warrants further exploration and could be due to self-selection into the study because they were experiencing ongoing symptoms, recall bias, external factors relating to the pandemic such as returning to school and exposure to other sources of infection, and the actual trajectory of the illness, although this wouldn't explain the high prevalence among test-negative CYP.

Second, symptoms reported at time of testing among test-positives were sore throat, headache, tiredness and loss of smell while test-negatives had sore throat, headache, fever and persistent cough. The symptom profile does not distinguish test-negatives and test-positives. However, the two groups could be separated according to the number of symptoms at three months, when $30.3 \%$ of test-positives and $16.2 \%$ of test-negatives had $3+$ symptoms, with tiredness and headache being common in both groups, but higher in the test-positives. Consideration of number of symptoms, rather than profile, is particularly important given that $53.3 \%$ of the test-negatives had at least one symptom 3 months post-test. These figures should be seen in the context of published norms. For example, high levels of fatigue have been reported in the general adolescent population with an estimated incidence of $30 \%$ in CYP aged $11-15$ over a 4-6-month pre-pandemic period ${ }^{22}$.

Third, our findings showed that, for both test-positives and test-negatives, those assigned to the latent class with "multiple symptoms" at three months were more likely to be female, older and have poorer physical and mental health before COVID-19, suggesting that pre-existing physical and mental health difficulties may influence symptoms at three months. Unsurprisingly, those with multiple physical 
symptoms had poorer mental health, reflecting the close relationship between physical and mental health.

Fourth, whilst the prevalence of physical symptoms differed between test-positives and test-negatives, no differences were found in mental health, wellbeing and fatigue scores. The scores on the measures are broadly comparable to pre-pandemic published norms on the standardised measures ${ }^{23,24}$ (https://sdqinfo.org/norms/UKNorm1.pdf). However, a large proportion ( 40\%) in both groups reported feeling worried, sad or unhappy. This is consistent with parent-reported surveys of mental health of CYP during the pandemic ${ }^{25}$ and highlights the need for mental health interventions ${ }^{26}$ for parents and CYP regardless of COVID status. The findings emphasise the importance of incorporating a comparator matched cohort of test-negative CYP who have contemporaneously experienced a pandemic, school closure and social isolation.

Despite a need to estimate Long COVID prevalence in CYP, this first requires an evidence-based case definition ${ }^{27}$. Our findings indicate that any definition of Long COVID should consider multiple symptoms and, as in adult studies on Long COVID, there may be different clusters of symptoms ${ }^{28}$. Given our findings of multiple, varied symptoms three months after infection, it seems a multi-component intervention will be required, building on existing interventions for management of problems such as headache and fatigue.

Our findings should be seen alongside the CYP literature. The commonest symptoms reported at three months in test-positives of tiredness, headache, shortness of breath, dizziness and anosmia are consistent with Molteni, et al. ${ }^{1}$ where parents reported symptoms $\geq 28$ days (fatigue $84 \%$, headache $80 \%$ and anosmia $80 \%$ ). They also reported that persistent symptom prevalence was higher in girls, teenagers and children with long-term conditions. The ONS survey ${ }^{6}$ describes population prevalence rates of persisting 'symptoms more than 4 weeks after COVID-19' whereas the present study gives rates in a testpositive population; hence apparent differences may be attributable to differences in definitions and methodology.

Taking the studies together, there is consistent evidence that some teenagers will have persisting symptoms after testing positive for SARS-CoV-2 and that mental and physical health symptoms are closely related. Avoiding false dichotomies between mind and body is likely to be helpful as, for example, stressed individuals may present with somatic symptoms or conversely persisting physical symptoms may be associated with depression and anxiety. Some individuals may develop somatic symptoms disorders ${ }^{29}$ and the existing evidence for effective management of conditions such as pain, headache and fatigue ${ }^{30}$ might be usefully evaluated in CYP presenting with persistent physical symptoms postCOVID. CYP with clinically impaired mental ill health should receive the appropriate evidence-based treatments whether or not they have physical symptoms. Family approaches and understanding of persistent symptoms is key ${ }^{31}$. Investigation of persisting symptoms may be needed or requested, with 
consideration of the potentially negative impact of protracted medical treatments or investigations if no abnormalities are found ${ }^{29}$.

This study has limitations. PCR-testing can result in some false negative and false positive results and we were unable to independently determine whether the test-negatives had previously had COVID unless they had been tested although this is likely to account for only a minority of cases. We could not recruit based on ethnicity as this was not recorded at time of test but ethnicity was very similar in test-positives and negatives (Table 2) and geographical region served as a proxy for socio-economic status; both these variables are thought to influence COVID in adults and could be important in Long COVID ${ }^{32}$. As in any self-selected online study, we need to acknowledge our response rate of $13.5 \%$. It is possible that there is a response bias for example, towards those continuing to experience symptoms at 3 months being more motivated to participate, resulting in an over-representation of symptom prevalence. It is also possible that recall bias influenced the reporting of symptoms at the time of testing as well as physical and mental health prior to testing, in particular, if tested positive. However, we tried to minimize the impact of this bias by only considering CYP that reported on baseline $\sim 3 \mathrm{~m}$ later. We did not assess whether symptoms were continuous for the entire 3 months, or whether they waxed and waned. Finally, the experiences of the CYP in January, February and March were likely to be highly varied with regard to school closure. At the time of testing, schools were closed, while, at 3 months after testing, schools had reopened albeit with social distancing, repeated testing and restriction of activities. Schools can be a source of both stress and support, and the return to school may partly explain some of the findings, in particular, the higher prevalence of symptoms at 3 months compared to baseline. The responders are largely representative of our target population though we have over-representation of girls and older CYP, with under-representation from North-West England and London. Inclusion of the comparator group was essential to place the findings within the wider context of the pandemic.

These data also reflect symptomatology at a time when the Alpha variant was predominant in the UK. Whilst these findings may change with different variants, the prospective nature of this study makes it uniquely placed to detect such changes across the pandemic waves.

In summary, post-COVID is different in CYP to adults and one should not extrapolate from the adult literature to decide policy and services for CYP. Our research demonstrates (1) the importance of having a control, test-negative group to interpret findings and prevalence estimates, (2) that it is essential to consider multiple symptoms in any clinical phenotype of Long COVID, (3) that mental and physical health symptoms should both be considered, (4) PCR-proven SARS-CoV-2 positive CYP had a higher frequency of any symptoms and multiple symptoms three months post-test than test-negatives. These results would have been very difficult to interpret without a control group and laboratory-confirmed SARS-COV-2 status. Hence, prevalence estimates and definitions of Long COVID should consider the presence of multiple symptoms, proven SARS-COV-2 status and include control groups. We will use these data and a Delphi consensus process to formulate a research definition of long COVID in CYP and will follow up the CYP over time to understand the long-term course in SARS-CoV-2 positive CYP. 
This paper does not provide an evidence base for management of Long COVID or for any infective/immunological mechanisms underlying it. More research is needed.

\section{Declarations}

Acknowledgement: Michael Lattimore, Public Health England, as Project Officer for the CLoCk study

\section{Author contributions:}

Terence Stephenson t.stephenson@ucl.ac.uk conceived the idea for the study, submitted the successful grant application and drafted the manuscript.

Snehal Pinto Pereira snehal.pereira@ucl.ac.uk conducted the statistical analyses for the manuscript and drafted the manuscript.

Roz Shafran r.shafran@ucl.ac.uk provided ideas on mental health to the original grant application, submitted the ethics and R\&D applications and drafted the manuscript.

Bianca De Stavola b.destavola@ucl.ac.uk provided statistical input to the design and conducted the analyses, including sample size calculations, and drafted the manuscript.

Natalia Rojas n.rojas@ucl.ac.uk supported the drafting of the manuscript and the statistical analyses.

Kelsey McOwat Kelsey.Mcowat@phe.gov.uk adapted the questionnaire for the online SNAP survey platform.

Ruth Simmons Ruth.Simmons@phe.gov.uk designed the participant sampling and dataflow.

Maria Zavala Maria.Zavala@phe.gov.uk undertook a separate pilot study which informed the CLoCk consent process and the online questionnaire.

Lauren O'Mahoney Ilom1@leicester.ac.uk contributed to the scoping review of the literature.

Trudie Chalder trudie.chalder@kcl.ac.uk contributed to the work on psychiatric liaison.

Esther Crawley, Esther.Crawley@bristol.ac.uk contributed to the work on chronic fatigue, contributed to and reviewed the manuscript

Tamsin Jane Ford tjf52@medschl.cam.ac.uk contributed to the work on epidemiology, schools and mental health and reviewed the manuscript

Anthony Harnden, anthony.harnden@phc.ox.ac.uk contributed to the work on primary care. 
Isobel Heyman i.heyman@ucl.ac.uk contributed to the work on psychiatric liaison and reviewed the manuscript.

Olivia Swann, Olivia.Swann@ed.ac.uk contributed to the work on infection and reviewed the manuscript.

Elizabeth Whittaker, e.whittaker@imperial.ac.uk contributed to the work on infection and reviewed the manuscript.

Shamez Ladhani shamez.ladhani@phe.gov.uk developed the study methodology, operationalised the regulatory and recruitment ideas for the study and revised the manuscript.

Olivia Swann and Elizabeth Whittaker designed the elements of the ISARIC Paediatric COVID-19 follow-up questionnaire which were incorporated into the online questionnaire used in this study to which all the CLoCk Consortium members contributed.

All members of the CLoCk Consortium made contributions to the conception or design of the work; were involved in drafting both the funding application and this manuscript; approved the version to be published; and agree to be accountable for all aspects of the work in ensuring that questions related to the accuracy or integrity of any part of the work are appropriately investigated and resolved.

\section{Additional Co-Applicants on the grant application and CLoCk Consortium members (alphabetical):}

Marta Buszewicz, University College London, m.buszewicz@ucl.ac.uk

Emma Dalrymple, PPI representative, emmajdalrymple@gmail.com

Shruti Garg, University of Manchester, Shruti.Garg@mft.nhs.uk

Dougal Hargreaves, Imperial College London, d.hargreaves@imperial.ac.uk

Michael Levin, Imperial College London, m.levin@imperial.ac.uk

Vanessa Poustie, University of Liverpool, v.poustie@liverpool.ac.uk

Terry Segal, University College London Hospitals NHS Foundation Trust, terry.segal@nhs.net

Malcolm Semple, University of Liverpool, M.G.Semple@liverpool.ac.uk

Kishan Sharma, Manchester University NHS Foundation Trust, Kishan.Sharma@mft.nhs.uk 
Competing interests statement: Terence Stephenson is Chair of the Health Research Authority and therefore recused himself from the Research Ethics Application.

Trudie Chalder is a member of the NICE committee for Long COVID. She has written self-help books on chronic fatigue and has conducted workshops on chronic fatigue and post infectious syndromes.

Data availability statement: All requests for data will be reviewed by the Children \& young people with Long Covid (CLoCk) study team, to verify whether the request is subject to any intellectual property or confidentiality obligations. Requests for access to the participant-level data from this study can be submitted via email to Clock@phe.gov.uk with detailed proposals for approval. A signed data access agreement with the CLoCK team is required before accessing shared data. Code is not made available as we have not used custom code or algorithms central to our conclusions.

Funding statement: Funded by The Department of Health and Social Care, in their capacity as the National Institute for Health Research (NIHR), and by UK Research \& Innovation (UKRI) who have awarded funding grant number COVLT0022. All research at Great Ormond Street Hospital NHS Foundation Trust and UCL Great Ormond Street Institute of Child Health is made possible by the NIHR Great Ormond Street Hospital Biomedical Research Centre. The views expressed are those of the author(s) and not necessarily those of the NHS, the NIHR or the Department of Health. SMP is supported by a UK Medical Research Council Career Development Award (ref: MR/P020372/1).

Study approved by Yorkshire \& The Humber - South Yorkshire Research Ethics Committee (REC reference: 21/YH/0060; IRAS project ID:293495).

\section{References}

1. Molteni, E., et al. Illness duration and symptom profile in symptomatic UK school-aged children tested for SARS-CoV-2. The Lancet Child \& Adolescent Health (2021).

2. Sudre, C.H., et al. Attributes and predictors of Long-COVID: analysis of COVID cases and their symptoms collected by the Covid Symptoms Study App. medRxiv (2020).

3. Amin-Chowdhury, Z. \& Ladhani, S.N. Causation or confounding: why controls are critical for characterizing long COVID. Nat Med(2021). 
4. Davis, H.E., et al. Characterizing long COVID in an international cohort: 7 months of symptoms and their impact. EClinicalMedicine, 101019 (2021).

5. Lopez-Leon, S., et al. More than 50 Long-term effects of COVID-19: a systematic review and metaanalysis. medRxiv (2021).

6. Office for National Statistics. Prevalence of ongoing symptoms following coronavirus (COVID-19) infection in the UK: 4 June 2021. (2021).

7. Pierce, M., et al. Mental health before and during the COVID-19 pandemic: a longitudinal probability sample survey of the UK population. The Lancet Psychiatry 7, 883-892 (2020).

8. Miller, F., et al. Prevalence of persistent symptoms in children during the COVID-19 pandemic: evidence from a household cohort study in England and Wales. medRxiv (2021).

9. Walsh-Messinger, J., et al. The Kids Are Not Alright: A Preliminary Report of Post-COVID Syndrome in University Students. medRxiv (2020).

10. Blankenburg, J., et al. Mental health of Adolescents in the Pandemic: Long-COVID19 or LongPandemic Syndrome? medRxiv, 2021.2005.2011.21257037 (2021).

11. Stephenson, T., et al. Long Covid and the mental and physical health of children and young people: national matched cohort (CLoCk) study protocol. BMJ Open (2021).

12. Ladhani, S.N., et al. SARS-CoV-2 infection and transmission in primary schools in England in JuneDecember, 2020 (sKIDs): an active, prospective surveillance study. The Lancet Child \& Adolescent Health 5, 417-427 (2021).

13. Sigfrid, L., et al. Addressing challenges for clinical research responses to emerging epidemics and pandemics: a scoping review. BMC Med 18, 190 (2020).

14. Ladhani, S.N., et al. COVID-19 in children: analysis of the first pandemic peak in England. Arch Dis Child 105, 1180-1185 (2020).

15. Waterfield, T., et al. Seroprevalence of SARS-CoV-2 antibodies in children: a prospective multicentre cohort study. Arch Dis Child (2020).

16. Goodman, R. Psychometric properties of the strengths and difficulties questionnaire. J Am Acad Child Adolesc Psychiatry 40, 1337-1345 (2001).

17. Tennant, R., et al. The Warwick-Edinburgh Mental Well-being Scale (WEMWBS): development and UK validation. Health Qual Life Outcomes 5, 63 (2007).

18. Wille, N., et al. Development of the EQ-5D-Y: a child-friendly version of the EQ-5D. Qual Life Res 19, 875-886 (2010).

19. Chalder, T., et al. Development of a fatigue scale. J Psychosom Res 37, 147-153 (1993).

20. McCutcheon, A.C. Latent class analysis, (Sage, Beverly Hills, CA, 1987).

21. Oehlert, G.W. A note on the delta method. American Statistician 46, 27-29 (1992).

22. Rimes, K.A., et al. Incidence, prognosis, and risk factors for fatigue and chronic fatigue syndrome in adolescents: a prospective community study. Pediatrics 119, e603-609 (2007). 
23. Cella, M. \& Chalder, T. Measuring fatigue in clinical and community settings. J Psychosom Res 69, 17-22 (2010).

24. Ng Fat, L., Scholes, S., Boniface, S., Mindell, J. \& Stewart-Brown, S. Evaluating and establishing national norms for mental wellbeing using the short Warwick-Edinburgh Mental Well-being Scale (SWEMWBS): findings from the Health Survey for England. Qual Life Res 26, 1129-1144 (2017).

25. Creswell, C., et al. Young people's mental health during the COVID-19 pandemic. The Lancet Child \& Adolescent Health (2021).

26. Kostyrka-Allchorne, K., et al. Supporting Parents \& Kids Through Lockdown Experiences (SPARKLE): A digital parenting support app implemented in an ongoing general population cohort study during the COVID-19 pandemic: A structured summary of a study protocol for a randomised controlled trial. Trials 22, 267 (2021).

27. Nesse, R.M. On the difficulty of defining disease: a Darwinian perspective. Med Health Care Philos 4, 37-46 (2001).

28. Whitaker, M., et al. Persistent symptoms following SARS-CoV-2 infection in a random community sample of 508,707 people. medRxiv (2021).

29. Willis, C. \& Chalder, T. Concern for Covid-19 cough, fever and impact on mental health. What about risk of Somatic Symptom Disorder? J Ment Health, 1-5 (2021).

30. Fisher, E., et al. Psychological therapies for the management of chronic and recurrent pain in children and adolescents. Cochrane Database Syst Rev 9, CD003968 (2018).

31. O'Connell, K., Berluti, K., Rhoads, S.A. \& Marsh, A.A. Reduced social distancing early in the COVID-19 pandemic is associated with antisocial behaviors in an online United States sample. PLoS One 16, e0244974 (2021).

32. Patel, P., Hiam, L., Sowemimo, A., Devakumar, D. \& McKee, M. Ethnicity and covid-19. BMJ 369, m2282 (2020).

\section{Tables}

Table 1. Response rate of participants who completed the 3-month questionnaire by sex, age and area of residence at time of testing, overall and stratified by SARS-CoV-2 status. 


\begin{tabular}{|c|c|c|c|c|}
\hline \multicolumn{2}{|l|}{ SARS-CoV-2 Status } & \multirow{2}{*}{$\begin{array}{c}\text { Target Population } \\
\text { N } \\
50,846\end{array}$} & \multirow{2}{*}{\multicolumn{2}{|c|}{$\begin{array}{rc}\mathrm{N} & \begin{array}{c}\text { Study Participants } \\
\text { \% of Target Population }\end{array} \\
& \\
6,804 & 13.4 \\
\end{array}$}} \\
\hline Overall & & & & \\
\hline \multirow[t]{14}{*}{ Negative } & & 27,798 & 3,739 & 13.5 \\
\hline & Female & 15,120 & 2,352 & 15.6 \\
\hline & Male & 12,678 & 1,387 & 10.9 \\
\hline & $11-15$ & 16,341 & 2,147 & 13.1 \\
\hline & $16-17$ & 11,457 & 1,592 & 13.9 \\
\hline & East Midlands & 2,132 & 340 & 15.9 \\
\hline & East of England & 4,278 & 630 & 14.7 \\
\hline & London & 5,356 & 629 & 11.7 \\
\hline & North East & 925 & 122 & 13.2 \\
\hline & North West & 3,816 & 426 & 11.2 \\
\hline & South East & 4,262 & 620 & 14.5 \\
\hline & South West & 1,554 & 293 & 18.9 \\
\hline & West Midlands & 3,414 & 431 & 12.6 \\
\hline & Yorkshire and the Humber & 2,061 & 248 & 12.0 \\
\hline \multirow[t]{3}{*}{ Positive } & & 23,048 & 3,065 & 13.3 \\
\hline & Female & 12,412 & 1,945 & 15.7 \\
\hline & Male & 10,636 & 1,120 & 10.5 \\
\hline \multirow[t]{2}{*}{ Age $(y)$} & $11-15$ & 13,630 & 1,721 & 26.0 \\
\hline & $16-17$ & 9,418 & 1,344 & 14.3 \\
\hline \multirow[t]{9}{*}{ Region } & East Midlands & 1,815 & 297 & 16.4 \\
\hline & East of England & 3,392 & 466 & 13.7 \\
\hline & London & 4,412 & 510 & 11.6 \\
\hline & North East & 819 & 111 & 13.6 \\
\hline & North West & 3,235 & 371 & 11.5 \\
\hline & South East & 3,496 & 483 & 13.8 \\
\hline & South West & 1,238 & 238 & 19.2 \\
\hline & West Midlands & 2,854 & 373 & 13.1 \\
\hline & Yorkshire and the Humber & 1,787 & 216 & 12.1 \\
\hline
\end{tabular}

Table 2. Frequencies (and percentages) of participants who completed the 3-month questionnaire by sex, age, ethnicity and area of residence at time of testing, overall and stratified by SARS-CoV-2 status. 


\begin{tabular}{|c|c|c|c|c|c|c|}
\hline & \multicolumn{2}{|c|}{ All participants } & \multicolumn{2}{|c|}{ SARS-CoV-2 Negative } & \multicolumn{2}{|c|}{ SARS-CoV-2 Positive } \\
\hline & $\mathbf{N}$ & $\%$ & $\mathbf{N}$ & $\%$ & $\mathbf{N}$ & $\%$ \\
\hline All & 6,804 & 100.0 & 3,739 & 100.0 & 3,065 & 100.0 \\
\hline Female & 4,297 & 63.2 & 2,352 & 62.9 & 1,945 & 63.5 \\
\hline \multicolumn{7}{|l|}{ Age (years) } \\
\hline $11-15$ & 3,868 & 56.8 & 2,147 & 57.5 & 1721 & 56.1 \\
\hline $16-17$ & 2,936 & 43.2 & 1,592 & 42.6 & 1,344 & 43.9 \\
\hline \multicolumn{7}{|l|}{ Ethnicity } \\
\hline White & 5,035 & 74.0 & 2,804 & 75.0 & 2,231 & 72.8 \\
\hline Asian/Asian British & 1,011 & 14.9 & 520 & 13.9 & 491 & 16.0 \\
\hline Mixed & 342 & 5.0 & 195 & 5.2 & 147 & 4.8 \\
\hline Black/African/Caribbean & 249 & 3.7 & 140 & 3.7 & 109 & 3.6 \\
\hline Other & 115 & 1.7 & 55 & 1.5 & 60 & 2.0 \\
\hline Unknown & 52 & 0.8 & 25 & 0.7 & 27 & 0.9 \\
\hline \multicolumn{7}{|l|}{ Region } \\
\hline East Midlands & 637 & 9.4 & 340 & 9.1 & 297 & 9.7 \\
\hline East of England & 1,096 & 16.1 & 630 & 16.9 & 466 & 15.2 \\
\hline London & 1,139 & 16.7 & 629 & 16.8 & 510 & 16.6 \\
\hline North East & 233 & 3.4 & 122 & 3.3 & 111 & 3.6 \\
\hline North West & 797 & 11.7 & 426 & 11.4 & 371 & 12.1 \\
\hline South East & 1,103 & 16.2 & 620 & 16.6 & 483 & 15.8 \\
\hline South West & 531 & 7.8 & 293 & 7.8 & 238 & 7.8 \\
\hline West Midlands & 804 & 11.8 & 431 & 11.5 & 373 & 12.2 \\
\hline Yorkshire and the Humber & 464 & 6.8 & 248 & 6.6 & 216 & 7.1 \\
\hline
\end{tabular}

Table 3. Number and percentage of reported symptom(s) at the time of test and at the 3 months questionnaire by SARS-CoV-2 status, overall and stratified by age-group. 


\begin{tabular}{|c|c|c|c|c|c|c|c|c|c|c|c|c|}
\hline & \multicolumn{4}{|c|}{ All participants } & \multicolumn{4}{|c|}{ Age: $11-15$} & \multicolumn{4}{|c|}{ Age: $16-17$} \\
\hline & \multicolumn{2}{|c|}{$\begin{array}{l}\text { SARS-CoV- } \\
2 \text { Negative }\end{array}$} & \multicolumn{2}{|c|}{$\begin{array}{l}\text { SARS-CoV- } \\
2 \text { Positive }\end{array}$} & \multicolumn{2}{|c|}{$\begin{array}{l}\text { SARS-CoV-2 } \\
\text { Negative }\end{array}$} & \multicolumn{2}{|c|}{$\begin{array}{c}\text { SARS-CoV-2 } \\
\text { Positive }\end{array}$} & \multicolumn{2}{|c|}{$\begin{array}{l}\text { SARS-CoV-2 } \\
\text { Negative }\end{array}$} & \multicolumn{2}{|c|}{$\begin{array}{l}\text { SARS- } \\
\text { CoV- } \\
2 \text { Positive }\end{array}$} \\
\hline & $\mathbf{N}$ & $\%$ & $\mathbf{N}$ & $\%$ & $\mathbf{N}$ & $\%$ & $\mathbf{N}$ & $\%$ & $\mathbf{N}$ & $\%$ & $\mathbf{N}$ & $\%$ \\
\hline 1 & 3,739 & 100.0 & 3,065 & 100.0 & 2,147 & 100.0 & 1,721 & 100.0 & 1,592 & 100.0 & 1,344 & 100.0 \\
\hline \multicolumn{13}{|l|}{ time of test } \\
\hline $\begin{array}{l}\text { o reported } \\
\text { rmptoms }\end{array}$ & 3,430 & 91.7 & 1,981 & 64.6 & 1,954 & 91.0 & 1,157 & 67.2 & 1,476 & 92.7 & 824 & 61.3 \\
\hline L-2 symptoms & 78 & 2.1 & 148 & & 59 & 2.8 & 93 & 5.4 & 19 & 1.2 & 55 & 4.1 \\
\hline 3-4 symptoms & 88 & 2.4 & 210 & 6.9 & 55 & 2.6 & 129 & 7.5 & 33 & 2.1 & 81 & 6.0 \\
\hline j+ symptoms & 143 & 3.8 & 726 & 23.7 & 79 & 3.7 & 342 & 19.9 & 64 & 4.0 & 384 & 28.6 \\
\hline \multicolumn{13}{|l|}{ Jecific symptoms } \\
\hline Fever & 148 & 4.0 & 548 & 17.9 & 91 & 4.2 & 271 & 15.8 & 57 & 3.6 & 277 & 20.6 \\
\hline Chills & 91 & 2.4 & 461 & 15.0 & 49 & 2.3 & 224 & 13.0 & 42 & 2.6 & 237 & 17.6 \\
\hline $\begin{array}{l}\text { Persistent } \\
\text { cough }\end{array}$ & 143 & 3.8 & 476 & 15.5 & 82 & 3.8 & 224 & 13.0 & 61 & 3.8 & 252 & 18.8 \\
\hline Tiredness & 125 & 3.3 & 696 & 22.7 & 71 & 3.3 & 331 & 19.2 & 54 & 3.4 & 365 & 27.2 \\
\hline $\begin{array}{l}\text { Shortness of } \\
\text { breath }\end{array}$ & 56 & 1.5 & 354 & 11.6 & 25 & 1.2 & 150 & 8.7 & 31 & 2.0 & 204 & 15.2 \\
\hline Loss of smell & 55 & 1.5 & 631 & 20.6 & 29 & 1.4 & 301 & 17.5 & 26 & 1.6 & 330 & 24.6 \\
\hline $\begin{array}{l}\text { Unusually } \\
\text { hoarse voice }\end{array}$ & 41 & 1.1 & 145 & 4.7 & 22 & 1.0 & 69 & 4.0 & 19 & 1.2 & 76 & 5.7 \\
\hline $\begin{array}{l}\text { Unusual } \\
\text { chest pain }\end{array}$ & 57 & 1.5 & 280 & 9.1 & 25 & 1.2 & 119 & 6.9 & 32 & 2.0 & 161 & 12.0 \\
\hline $\begin{array}{l}\text { Unusual } \\
\text { abdominal } \\
\text { pain }\end{array}$ & 44 & 1.2 & 138 & 4.5 & 27 & 1.3 & 71 & 4.1 & 17 & 1.1 & 67 & 5.0 \\
\hline Diarrhoea & 41 & 1.1 & 166 & 5.4 & 24 & 1.1 & 71 & 4.1 & 17 & 1.1 & 95 & 7.1 \\
\hline Headaches & 178 & 4.8 & 806 & 26.3 & 108 & 5.0 & 417 & 24.2 & 70 & 4.4 & 389 & 28.9 \\
\hline $\begin{array}{l}\text { Confusion, } \\
\text { disorientation } \\
\text { or } \\
\text { drowsiness }\end{array}$ & 29 & 0.8 & 225 & 7.3 & 11 & 0.5 & 91 & 5.3 & 18 & 1.1 & 134 & 10.0 \\
\hline $\begin{array}{l}\text { Unusual eye- } \\
\text { soreness }\end{array}$ & 30 & 0.8 & 185 & 6.0 & 16 & 0.8 & 83 & 4.8 & 14 & 0.9 & 102 & 7.6 \\
\hline $\begin{array}{l}\text { Skipping } \\
\text { meals }\end{array}$ & 67 & 1.8 & 360 & 11.8 & 32 & 1.5 & 159 & 9.2 & 35 & 2.2 & 201 & 15.0 \\
\hline $\begin{array}{l}\text { Dizziness or } \\
\text { light- } \\
\text { headedness }\end{array}$ & 86 & 2.3 & 462 & 15.1 & 43 & 2.0 & 206 & 12.0 & 43 & 2.7 & 256 & 19.1 \\
\hline Sore throat & 200 & 5.4 & 687 & 22.4 & 122 & 5.7 & 351 & 20.4 & 78 & 4.9 & 336 & 25.0 \\
\hline $\begin{array}{l}\text { Unusual } \\
\text { strong } \\
\text { muscle pains }\end{array}$ & 45 & 1.2 & 338 & 11.0 & 22 & 1.0 & 154 & 9.0 & 23 & 1.4 & 184 & 13.7 \\
\hline
\end{tabular}


$\begin{array}{lllllllllllll}\text { Earache or } & 41 & 1.1 & 155 & 5.1 & 16 & 0.8 & 69 & 4.0 & 25 & 1.6 & 86 & 6.4\end{array}$ ringing in ears
Raised welts
$\begin{array}{lll}7 & 0.2 & 35\end{array}$
$35 \quad 1.1$
$\begin{array}{llll}.1 & 5 & 0.2 & 19\end{array}$
1.12
0.1
16
1.2

on skin or

swelling

Red/purple

sores/blisters

on feet
9
$\begin{array}{lll}0.2 & 21 & 0.7\end{array}$
$\begin{array}{lll}8 & 0.4 & 12\end{array}$
$0.7 \quad 1$
$0.1 \quad 9$
0.7

on feet

$\begin{array}{lllll}17 & 0.5 & 73 & 2.4 & 13\end{array}$

$0.6 \quad 38$

2.2

4

$0.3 \quad 35$

2.6

Other

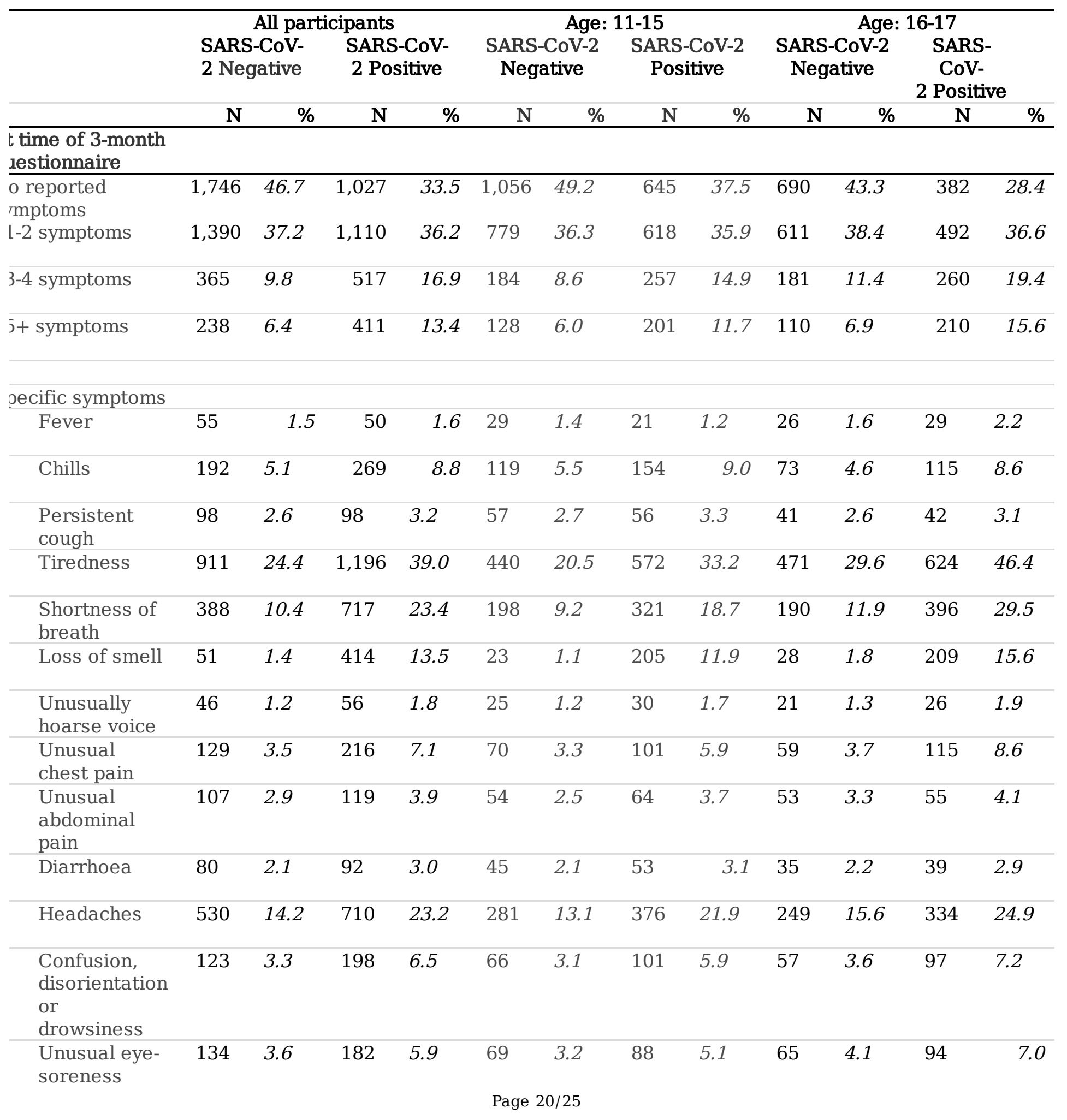




\begin{tabular}{|c|c|c|c|c|c|c|c|c|c|c|c|c|}
\hline $\begin{array}{l}\text { Skipping } \\
\text { meals }\end{array}$ & 275 & 7.4 & 296 & 9.7 & 132 & 6.2 & 135 & 7.8 & 143 & 9.0 & 161 & 12.0 \\
\hline $\begin{array}{l}\text { Dizziness or } \\
\text { light- } \\
\text { headedness }\end{array}$ & 314 & 8.4 & 419 & 13.7 & 169 & 7.9 & 202 & 11.7 & 145 & 9.1 & 217 & 16.2 \\
\hline Sore throat & 281 & 7.5 & 291 & 9.5 & 147 & 6.9 & 181 & 10.5 & 134 & 8.4 & 110 & 8.2 \\
\hline $\begin{array}{l}\text { Unusual } \\
\text { strong } \\
\text { muscle pains }\end{array}$ & 83 & 2.2 & 165 & 5.4 & 45 & 2.1 & 77 & 4.5 & 38 & 2.4 & 88 & 6.6 \\
\hline $\begin{array}{l}\text { Earache or } \\
\text { ringing in } \\
\text { ears }\end{array}$ & 165 & 4.4 & 191 & 6.2 & 96 & 4.5 & 108 & 6.3 & 69 & 4.3 & 83 & 6.2 \\
\hline $\begin{array}{l}\text { Raised welts } \\
\text { on skin or } \\
\text { swelling }\end{array}$ & 32 & 0.9 & 48 & 1.6 & 16 & 0.8 & 28 & 1.6 & 16 & 1.0 & 20 & 1.5 \\
\hline $\begin{array}{l}\text { Red/purple } \\
\text { sores/blisters } \\
\text { on feet }\end{array}$ & 40 & 1.1 & 35 & 1.1 & 23 & 1.1 & 21 & 1.2 & 17 & 1.1 & 14 & 1.0 \\
\hline Other & 590 & 15.8 & 335 & 10.9 & 369 & 17.2 & 199 & 11.6 & 221 & 13.9 & 136 & 10.1 \\
\hline
\end{tabular}

Table 4. Characteristics of CYP assigned to each latent class, by SARS-CoV-2 status 


\begin{tabular}{|c|c|c|c|c|}
\hline & \multicolumn{2}{|c|}{ SARS-CoV-2 test Negatives $(\mathrm{N}=3,739)$} & \multicolumn{2}{|c|}{ SARS-CoV-2 test Positives $(\mathrm{N}=3,065)$} \\
\hline & Class 1 & Class 2 & Class 1 & Class 2 \\
\hline & $\%$ & $\%$ & $\%$ & $\%$ \\
\hline $\begin{array}{l}\text { All } \\
\text { Baseline }\end{array}$ & 80.7 & 19.3 & 70.4 & 29.6 \\
\hline \multicolumn{5}{|l|}{ Sex } \\
\hline Male & 88.5 & 11.5 & 82.4 & 17.6 \\
\hline Female & 79.0 & 21.0 & 65.9 & 34.1 \\
\hline \multicolumn{5}{|l|}{ Age (years) } \\
\hline 11-15 & 84.0 & 16.0 & 75.3 & 24.7 \\
\hline $16-17$ & 80.6 & 19.4 & 67.7 & 32.3 \\
\hline \multicolumn{5}{|l|}{ Previous physical health } \\
\hline Very poor/poor/OK & 71.9 & 28.1 & 62.8 & 37.2 \\
\hline Good/v Good & 85.8 & 14.2 & 74.8 & 25.2 \\
\hline \multicolumn{5}{|l|}{ Previous mental health } \\
\hline Very poor/poor/OK & 72.3 & 27.7 & 60.4 & 39.6 \\
\hline Good/v Good & 88.8 & 11.2 & 79.2 & 20.8 \\
\hline \multicolumn{5}{|l|}{ At 3 months } \\
\hline \multicolumn{5}{|l|}{ EQ5DY*: } \\
\hline Mobility & 62.0 & 38.0 & 37.5 & 62.5 \\
\hline Self-care & 62.2 & 37.9 & 40.3 & 59.7 \\
\hline Usual activities & 59.2 & 40.8 & 40.3 & 59.7 \\
\hline Pain/discomfort & 50.2 & 49.8 & 35.4 & 64.6 \\
\hline Feeling worried & 69 & 31 & 55.4 & 44.6 \\
\hline \multicolumn{5}{|l|}{ SDQ total difficulties } \\
\hline $\begin{array}{l}\text { Median } \\
\left(25^{\text {th }}, 75^{\text {th }}\right)\end{array}$ & $\begin{array}{c}10 \\
(6,14)\end{array}$ & $\begin{array}{c}16 \\
(11,20)\end{array}$ & $\begin{array}{c}9 \\
(6,14)\end{array}$ & $\begin{array}{c}15 \\
(10,19)\end{array}$ \\
\hline \multicolumn{5}{|l|}{ SWEMBS } \\
\hline $\begin{array}{l}\text { Median } \\
\left(25^{\text {th }}, 75^{\text {th }}\right)\end{array}$ & $\begin{array}{c}21.5 \\
(19.3,24.1)\end{array}$ & $\begin{array}{c}18.6 \\
(16.9,21.5)\end{array}$ & $\begin{array}{c}21.5 \\
(19.3,25.0)\end{array}$ & $\begin{array}{c}19.3 \\
(17.4,22.4)\end{array}$ \\
\hline Mean (SD) & $21.9(4.4)$ & $19.1(3.7)$ & $22.1(4.3)$ & $20.0(3.9)$ \\
\hline \multicolumn{5}{|l|}{ Chalder fatigue scale } \\
\hline $\begin{array}{l}\text { Median } \\
\left(25^{\text {th }}, 75^{\text {th }}\right)\end{array}$ & $\begin{array}{c}11 \\
(10,14)\end{array}$ & $\begin{array}{c}16 \\
(12,20)\end{array}$ & $\begin{array}{c}11 \\
(11,14)\end{array}$ & $\begin{array}{c}17 \\
(13,21)\end{array}$ \\
\hline Mean (SD) & $11.6(4.5)$ & $16.4(6.0)$ & $11.8(4.2)$ & $17.0(5.7)$ \\
\hline
\end{tabular}

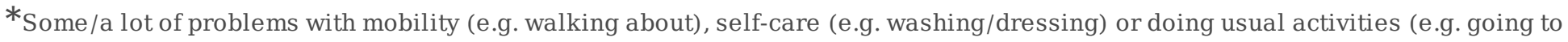
school); some/a lot of pain/discomfort or a bit/very worried, sad or unhappy.

SD: standard deviation

Figures 


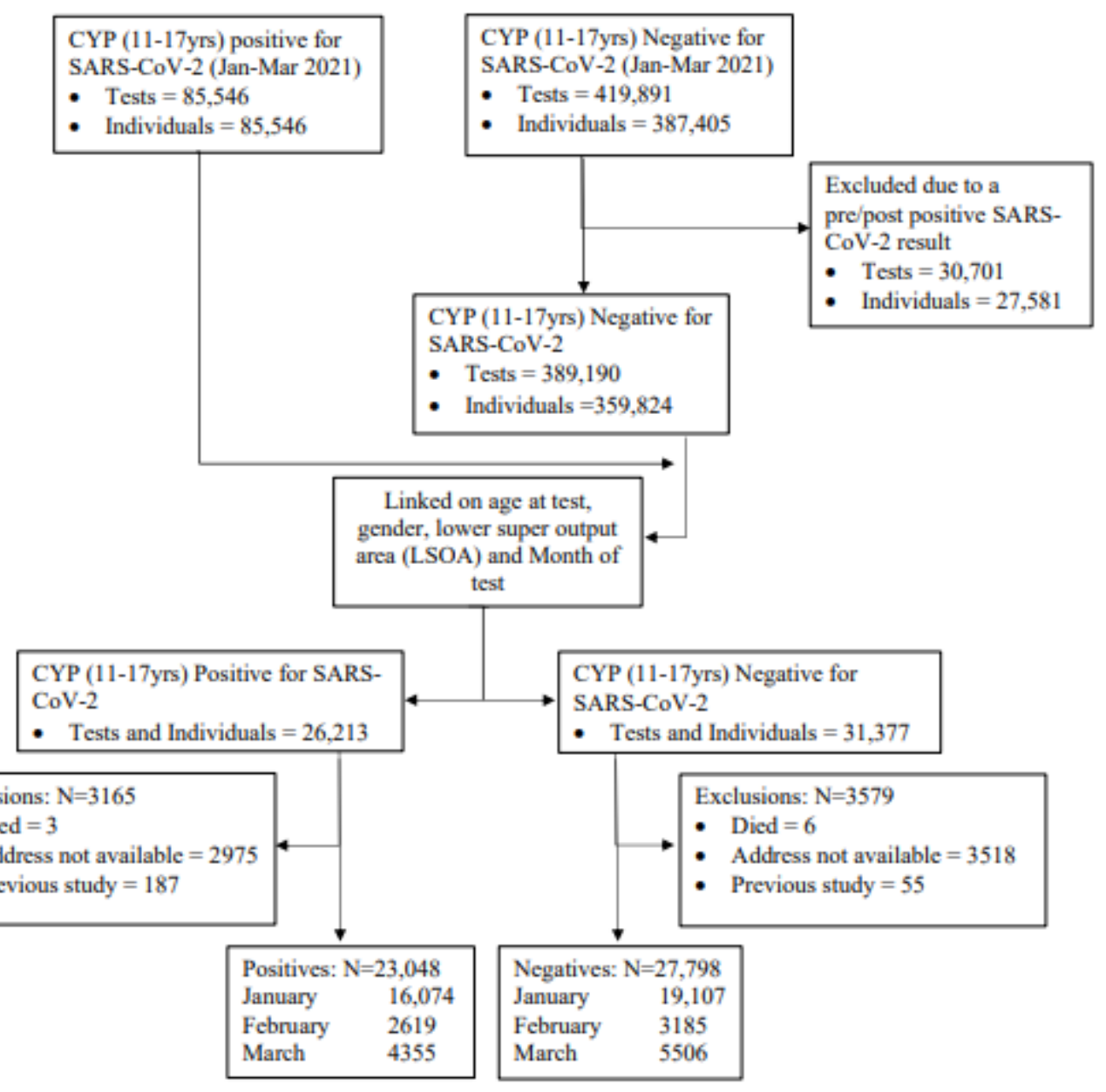

\section{Figure 1}

Flowchart of young people invited to participate in the Study. 
(a) Positives
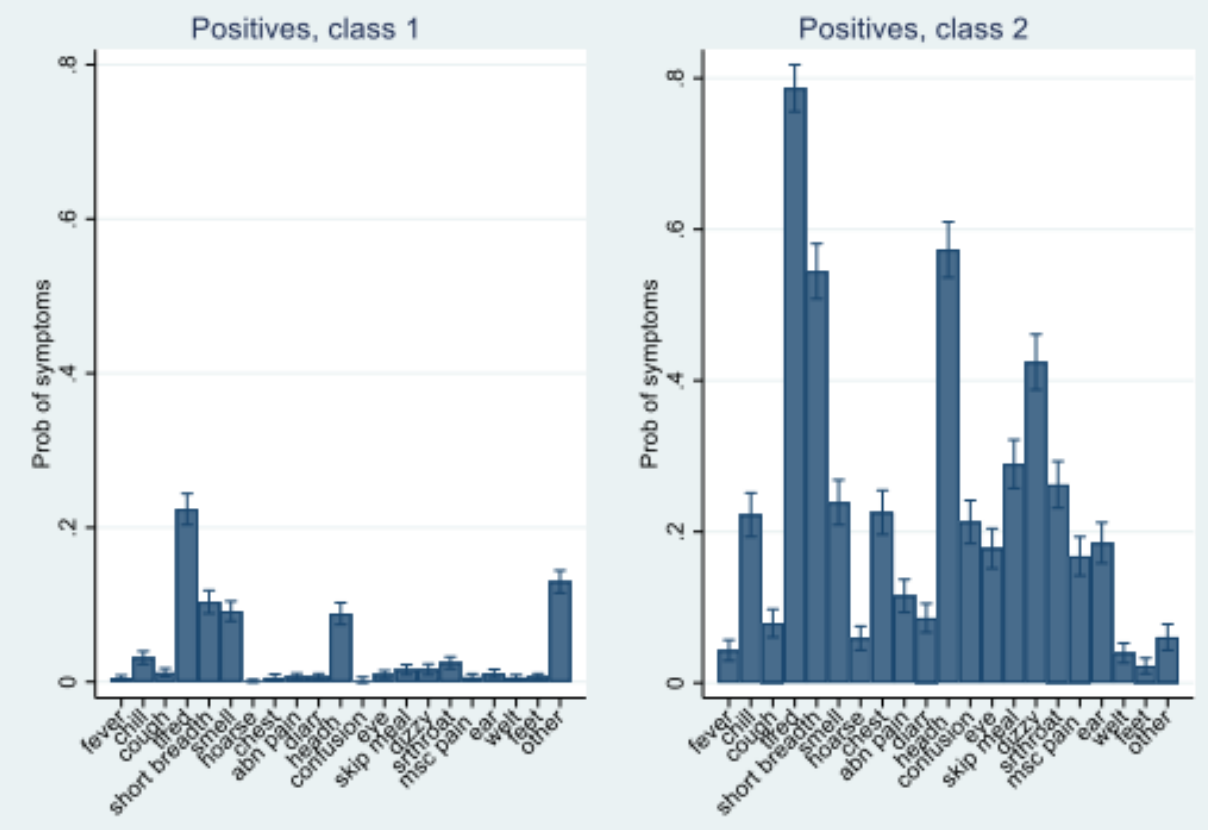

(b) Negatives
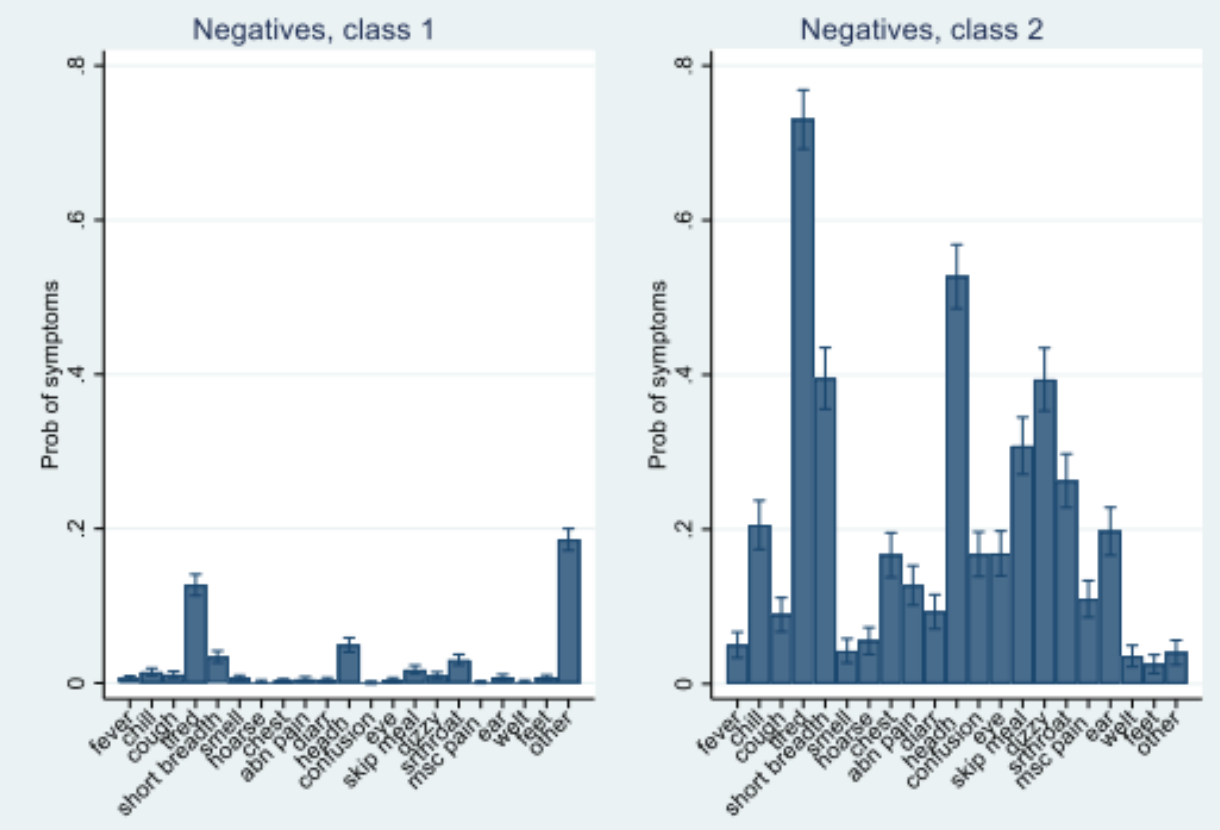

\section{Figure 2}

(a) Latent classes of symptoms at 3 months in SARS-CoV-2 test positive CYP; (b) Latent classes of symptoms at 3 months in SARS-CoV-2 test negative CYP. BIC for 1-class solution for the two test groups: 66,743.600; BIC for 2-class solution for the two test groups: 58,991.627; no convergence for the 3-class model 


\section{Supplementary Files}

This is a list of supplementary files associated with this preprint. Click to download.

- SupplementaryInformationCLoCk3monthpaper.pdf 